\title{
Characterization of Grain Structure and Porosity in Selective Laser Melted Cu- 4.3Sn for Enhancing Electrical Connector Fabrication
}

\author{
Anthony P. Ventura ${ }^{1}$, C. Austin Wade ${ }^{1}$, Wojciech Z. Misiolek ${ }^{1}$, Masashi Watanabe ${ }^{1}$, \\ Greg Pawlikowski ${ }^{2}$, Martin Bayes ${ }^{2}$ \\ 1. Lehigh University, Materials Science and Engineering Department, Bethlehem, PA, USA \\ 2. TE Connectivity, Harrisburg, PA, USA
}

Copper $(\mathrm{Cu})$ has the highest electrical conductivity of any commercial metal; it is surpassed only by silver, which is prohibitively expensive for use in commercial applications. Worldwide, up to $65 \%$ of $\mathrm{Cu}$ and its alloys produced are destined for electrical applications [1]. Although $\mathrm{Cu}$ and its alloys have seen extensive use in the development of additive manufacturing (AM) technology, they often assume the role of a binding or infiltration phase rather than the primary material. Developments in selective laser melting (SLM) have allowed for the fabrication of relatively high density $\mathrm{Cu}$ alloy components with unique microstructures which opens up the possibility of using AM for the production of electronic components. However, the expansion of AM into electronic applications requires an understanding of microstructure development during fabrication of $\mathrm{Cu}$ alloys by SLM. Although grain structure has been characterized in some titanium, aluminum, and ferrous alloys, there has been limited work characterizing $\mathrm{Cu}$ alloys fabricated by SLM [2-4]. In this study the microstructure of a $\mathrm{Cu}-4.3 \mathrm{Sn}$ alloy fabricated by SLM was investigated using scanning electron microscopy (SEM) and aberrationcorrected scanning transmission electron microscopy (STEM).

Pre-alloyed $\mathrm{Cu}-4.3 \mathrm{Sn}$ powder produced by air atomization was supplied by Ecka Granules with a maximum particle size of 63 microns. A 200 W EOSINT M 280 direct metal laser sintering unit at TE Connectivity was used for the fabrication of all samples. The laser parameters were optimized for a maximum attainable density of approximately $96 \%$ before $10 \mathrm{~mm}^{3}$ cubes were fabricated using a simple raster scan strategy rotated $30^{\circ}$ between each build layer. Strip samples were also prepared to measure electrical conductivity via the 4 point probe technique. Metallography was performed using standard preparation techniques and samples were etched in Klemm's I for observation in polarized light optical microscopy. Metallographic samples were then re-polished and used for electron backscatter diffraction (EBSD) conducted on a Hitachi 4300 field emission SEM. TEM specimens were prepared by a Focused Ion-Beam (FIB) instrument on an FEI SCIOS dual-beam instrument and final thinning was performed on a Fischione 1040 Nanomill. TEM/STEM analysis was completed using a JEOL JEM-ARM200CF aberration-corrected STEM.

The polarized optical micrographs in Figure 1 show weld pools characteristic to the SLM process. Micro-voiding and a unique substructure can be seen at the weld pool interfaces during backscattered electron SEM observation. Grains were found to be elongated along the build direction, often spanning multiple build layers. Figure 2 is EBSD inverse pole figure maps perpendicular to the build direction showing grain orientation in dense and porous regions. Additionally, grain texture was observed along the build height of the entire sample in the transverse direction. High-angle annular dark-field STEM imaging was used along with X-ray energy dispersive spectrometry to characterize the weld pool interfaces and identify second phase particles within the microstructure. 


\section{References}

[1] J.R. Davis in "Copper and Copper Alloys: ASM Specialty Handbook", ed. 1 (ASM International, Materials Park) p. 3.

[2] L. Thijs et al., Acta Materialia 58 (2010), p. 3303.

[3] L. Thijs et al., Acta Materialia 61 (2013), p. 1809.

[4] T. Niendorf et al., Metallurgical and Materials Transactions 44B (2013), p. 794.

[5] The authors would like to acknowledge the joint funding from the Research for Advanced Manufacturing in Pennsylvania program and TE Connectivity, Ltd.
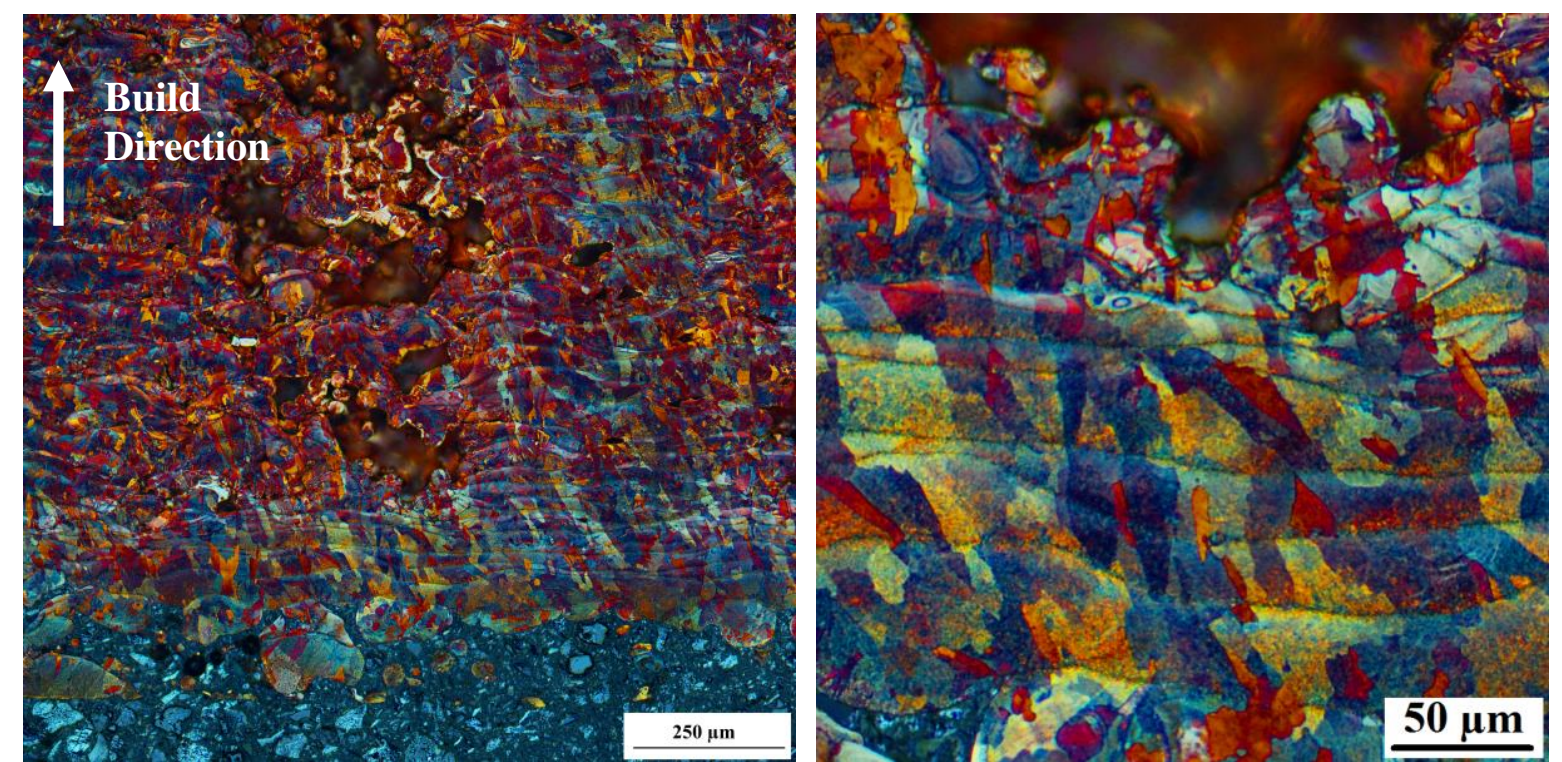

Figure 1. Polarized micrographs of $\mathrm{Cu} 4.3 \mathrm{Sn}$ etched in Klemm's I. Mounting material visible in bottom of left micrograph.
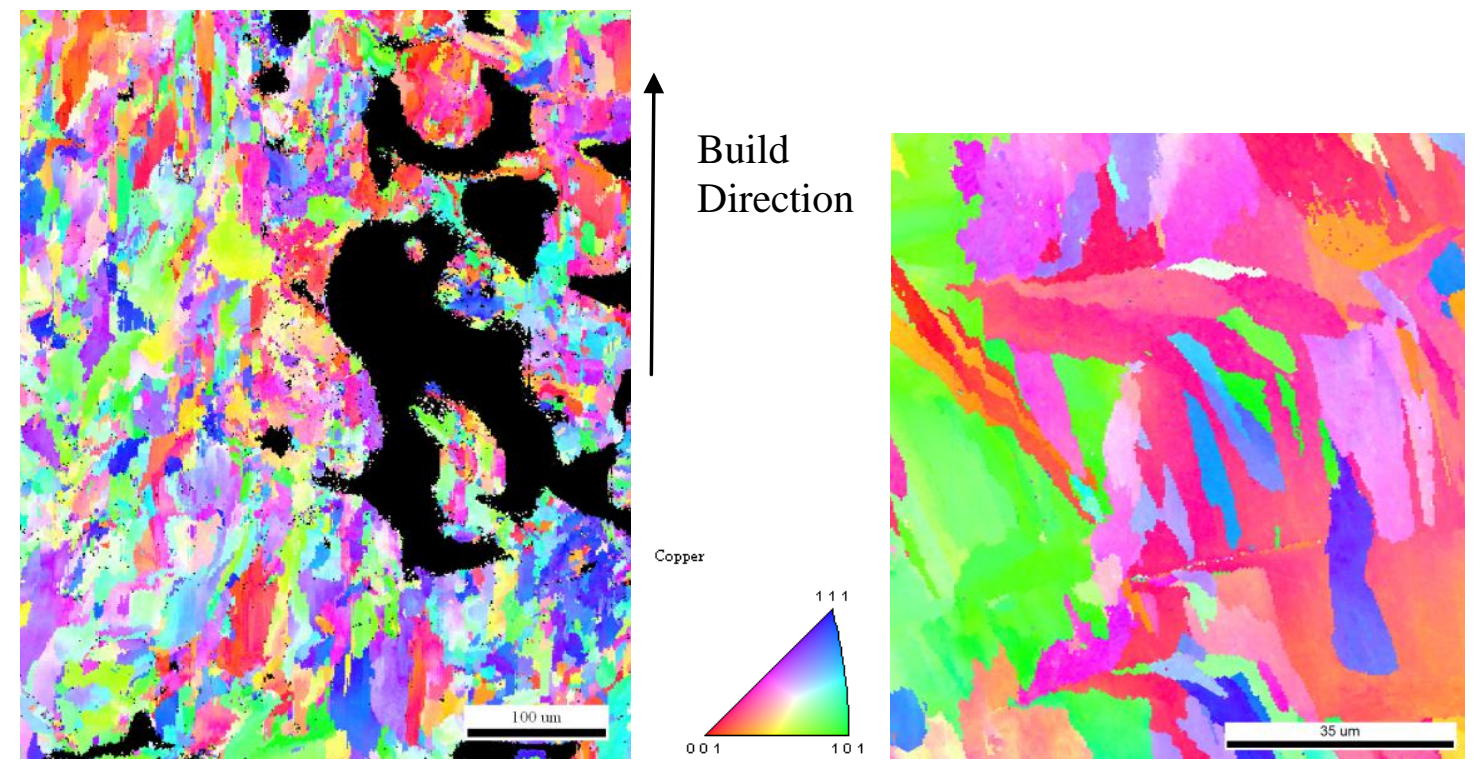

Figure 2. Inverse pole figure map of SLM Cu-4.3Sn at low magnification (left) and high magnification of a dense area (right). Black areas are porosity. 